\title{
Multi-omics analysis to identify driving factors in colorectal cancer
}

\author{
Xi Xu $u^{\ddagger}, 1$ (D) Chaoju Gong ${ }^{\ddagger, 2}$, Yunfeng Wang ${ }^{3}$, Yanyan $\mathrm{Hu}^{4}$, Hong Liu**,5,6 \& Zejun \\ Fang*,4,7 iD \\ ${ }^{1}$ Department of Pathology, The Second Affiliated Hospital, Zhejiang University School of Medicine, Hangzhou, 310009, China \\ ${ }^{2}$ Central Laboratory, The Municipal Affiliated Hospital of Xuzhou Medical University, Xuzhou, 221106, PR China \\ ${ }^{3}$ Institute for Integrative Biology of the Cell, UMR 9198, CNRS, Commissariat à I'Energie Atomique et aux Énergies Alternatives \\ (CEA), Université Paris-Sud, 91198 Gif-sur-Yvette, Palaiseau, 91120, France \\ ${ }^{4}$ Central Laboratory, Sanmen People's Hospital of Zhejiang Province, Sanmen, 317100, PR China \\ ${ }^{5}$ Zhejiang Normal University - Jinhua People's Hospital Joint Center for Biomedical Research, Jinhua, 321004, PR China \\ ${ }^{6}$ The Affiliated Hospital of Jinhua Polytechnic College, Jinhua, 321000, PR China \\ ${ }^{7}$ Central Laboratory, Sanmenwan Branch, The First Affiliated Hospital, College of Medicine, Zhejiang University, Sanmen, 317100 , \\ PR China \\ *Author for correspondence: Tel./Fax: +86 5768337 8395; fang_zj@126.com \\ **Author for correspondence: Tel./Fax: +86 5798913 8696; Liu_Hong@zju.edu.cn \\ $\ddagger$ Authors contributed equally
}

\begin{abstract}
Aim: We aim to identify driving genes of colorectal cancer (CRC) through multi-omics analysis. Materials \& methods: We downloaded multi-omics data of CRC from The Cancer Genome Atlas dataset. Integrative analysis of single-nucleotide variants, copy number variations, DNA methylation and differentially expressed genes identified candidate genes that carry CRC risk. Kernal genes were extracted from the weighted gene co-expression network analysis. A competing endogenous RNA network composed of CRCrelated genes was constructed. Biological roles of genes were further investigated in vitro. Results: We identified $L R R C 26$ and REP15 as novel prognosis-related driving genes for CRC. $L R R C 26$ hindered tumorigenesis of CRC in vitro. Conclusion: Our study identified novel driving genes and may provide new insights into the molecular mechanisms of CRC.
\end{abstract}

First draft submitted: 22 February 2020; Accepted for publication: 8 June 2020; Published online: 23 June 2020

Keywords: ceRNA network • colorectal cancer • driving genes • LRRC26 • multi-omics analysis • REP15

Colorectal cancer (CRC) is one of the most frequently occurring carcinomas and has become a major cause of cancerrelated mortality worldwide [1]. In China, approximately 370,000 cases of CRC are diagnosed and 180,000 patients die every year from CRC, ranking CRC as third in cancer morbidity and fifth in cancer mortality [2]. Although surgical excision and chemotherapy can achieve a substantial improvement in the CRC prognosis, approximately a third of CRC patients are diagnosed at an advanced tumor stage, and the 5-year survival rate is only slightly greater than $10 \%$ [3]. Hence, it is of great value to develop CRC-specific biomarkers for early diagnosis.

Tumor progression is often accompanied by gene mutations at the transcriptional or post-transcriptional levels; therefore, the identification of genetic and epigenetic variations would help to promote diagnosis efficiency [4]. Among epigenetic alterations, single-nucleotide variants ( $\mathrm{SNVs}$ ), copy number variations (CNVs) and DNA methylation (MET) are critical for tumorigenesis [5-7]. The continuous improvement of public databases provides a source of available sequence and array data, which enables the identification of various types of candidate biomarkers. Several studies have illustrated that multi-omics analysis of the variations in genomics, epigenomics and transcriptomics is important for cancer-related biomarker identification $[8,9]$.

In this study, we identified CRC-specific candidate biomarkers by applying multi-omics analysis. We identified SNVs, CNVs, differential DNA-METs and differentially expressed genes (DEGs). After comprehensive analysis, we constructed a multidimensional landscape of CRC-specific genes via correlation analysis and combined these genes with clinicopathological information to identify candidate genes for cancer subtype classification. In addition, 
we constructed a noncoding RNA regulation network for candidate genes according to the competing endogenous RNA (ceRNA) hypothesis. As a result, we identified several biomarkers for use in CRC diagnosis and prognosis; these may lead to useful insights for CRC tumorigenesis.

\section{Materials \& methods}

Data download \& preprocessing

The somatic mutation information (reads were aligned against the human genome [hg19]), copy number segment information (Affymetrix genome-wide human SNP array 6.0), methylation information (platform: Illumina 450K DNA methylation array) and mRNA expression profile data related to CRC and the relevant clinical data were obtained from the The Cancer Genome Atlas dataset (TCGA) database. Considered that array is dependent upon the signals generated via hybridization, the sensitivity for score in gene expression could not reach to the levels of RNA-sequence, the score of which shows directly expressing levels of a specific mRNA, we exploited RNA-sequence data from TCGA in this study. A total of 367 samples had multiple sets of data with somatic mutations, CNVs, methylations and mRNA expression profiles. The follow-up analysis was based on these 367 samples.

\section{SNV analysis}

We exploited MutSigCV to analyze significant somatic variations for the reason that MutSigCV considers tumor heterogeneity and looks for high frequency mutations relative to background mutations. Thus, MutSigCV builds a mutation background model based on the mutation information, and judges whether the mutation of each gene is more significant than the accidental mutation according to the model [10]. The R packages maftools [11] and SomaticSignatures [12] were used to analyze the mutation characteristic tags of CRC samples. According to each tag, unsupervised hierarchical clustering was carried out to identify the clinical characteristics of different subgroups with different mutation tags.

\section{CNV analysis}

GISTIC 2.0 was used to identify the region of CNV. The parameters were set as follows: $Q \leq 0.05$ was the change significance standard; a confidence level of 0.95 was applied to determine the peak interval; and the relative length of chromosome was $\geq 0.98$ to analyze horizontal variation of the chromosome arm. The corresponding GISTIC module in GenePattern was also used for analysis.

\section{DNA methylation analysis}

Level 3 DNA methylation data was downloaded from TCGA. Absent loci were removed to obtain the spectrum of methylation levels related to CRC, and the R package LIMMA [13] was used to identify differentially methylated sites with $\mathrm{p} \leq 0.05$ and $\mid \log$ fold change $(\mathrm{FC}) \mid \geq 0.4$. At last, differentially methylated sites were mapped to the corresponding genes for further study.

\section{Identification of differential expression}

RNA expression profile data (level 3) of CRC and adjacent normal tissues were obtained from TCGA. LIMMA was used to identify DEGs in the normal and CRC tissues. Genes with $\log 2|\mathrm{FC}| \geq 1$ and $\mathrm{p} \leq 0.05$ were identified as DEGs. We also employed LIMMA to analyze differentially expressed microRNAs (DEmicroRNAs) and differentially expressed lncRNAs (DElncRNAs), applying a threshold of $\mathrm{p} \leq 0.05$ and $\log 2|\mathrm{FC}| \geq 1$.

\section{WGCNA analysis}

Weighted gene co-expression network analysis (WGCNA) is a systematic biological method that focuses on the relationships of patterns and gene expression profiles in a scale-free network [14]. The R package WGCNA was used for the co-expression network analysis using the expression values of the genes. First, a similarity matrix of gene expression was constructed, and a suitable soft threshold $\beta$ was selected to ensure a scale-free network. Second, the expression matrix was transformed into an adjacency matrix and then into a topology matrix. Based on topological overlap measure, we clustered the genes into different modules through average linkage hierarchical clustering analysis. At last, we calculated the eigenvector value of each module in turn and assessed the correlation between modules and phenotypes. We considered modules with the highest correlation as candidates for further study. 


\section{Gene set enrichment analysis}

Gene set enrichment analysis (GSEA) was employed to explore the potentially enriched signaling pathways [15]. Given that some gene sets related to noncanonical cancer-related pathways are present in C2 but are absent in C6, so $\mathrm{C} 2$ is more comprehensive to avoid missed detection. Hence, we chose the data set c2.cp.kegg. v6.0 symbols.gmt as the reference gene set. Normalized $\mathrm{p} \leq 0.05$ and $\mid$ normalized enrichment score $\mid \geq 1$ were set as the cut-off criteria.

\section{Immunohistochemistry}

A total of 172 human CRC samples and their paired adjacent normal tissues were collected from the Sanmen People's Hospital of Zhejiang Province. All samples were obtained with the approval from the Institutional Ethics Committee, informed written consent was obtained and the study was performed with the approval of the Ethics Committee of Sanmen People's Hospital of Zhejiang Province. Immunohistochemistry was performed by an EnVision Detection System (DAKO, CA, USA) according to the manufacturer's instruction. Briefly, paraffinembedded tissue sections ( $4 \mu \mathrm{m}$ thick) were treated with routine dewaxing and hydration. Antigen in the specimens was retrieved according to the immunohistochemistry kits prior to testing. The samples were blocked with $10 \%$ normal goat serum for $30 \mathrm{~min}$ and then incubated with primary antibodies overnight at $4{ }^{\circ} \mathrm{C}$. The following day, the tissue samples were washed three-times with $0.1 \mathrm{M}$ phosphate-buffered saline $(\mathrm{pH}=7.4)$ and then underwent $\mathrm{DAB}$ staining, hematoxylin staining and neutral balata fixation. The antibodies employed in these assays were as follows: REP15 (1:25, Abcam, Cambridge, UK), LRRC26 (1:200, GeneTex, SA, USA), E-Cadherin (1:200, Abcam, Cambridge, UK) and vimentin (1:200, Abcam, Cambridge, UK).

Each specimen was scored as follows: if positive cells accounts for $0-5 \%$ positive cells, the specimen was scored as 0 ; specimen with $5-25 \%$ positive cells was scored as 1 ; specimen with $26-50 \%$ positive cells was scored as 2 ; specimen with $51-75 \%$ positive cells was scored as 3 and specimen with over $75 \%$ positive cells was scored as 4. The staining intensities were graded from 0-3: 0 represents for no staining, 1 represents for weak staining, 2 represents for moderate staining, and 3 represents for strong staining. Subsequently, two investigators assessed and confirmed the staining results independently. Immunohistochemistry score equals the percentage of positive cells multiplied the staining intensity, and we regarded scores from 0 to 6 as low expression or scores from 8 to 12 as high expression. Cases with a discrepancy in score were discussed to obtain a consensus.

\section{Reagents \& materials}

Cell-culture reagents were purchased from Gibco (NE, USA). Lipofectamine was obtained from Invitrogen (CA, USA). Secondary antibodies for western blotting were purchased from Li-COR Biosciences (NE, USA). The pCMV3 plasmid containing full length clone DNA of human LRRC26 was obtained from Sino Biological (Beijing, China) and a pLKO.1 plasmid with an shRNA targeting AGCACTGGCACCAGGGACTTT of human LRRC26 coding sequence was obtained from Sigma-Aldrich (MO, USA). All transfections were performed with Lipofectamine 2000 (Invitrogen) according to the manufacturer's instructions.

\section{Cell lines \& cultures}

The CRC cell lines SW480, LoVo, HCT116, SW620, HT29 and Rko were purchased from the American Type Culture Collection (USA) and cultured in RPMI 1640 supplemented with $10 \%$ fetal bovine serum (Invitrogen). All cell lines were maintained in a $5 \% \mathrm{CO}_{2}$ atmosphere at $37^{\circ} \mathrm{C}$. Certifications for cell lines were shown in Supplementary data $1-6$.

\section{Western blot}

Cells were lysed with RIPA buffer (Beyotime, China) to extract protein. The concentration of protein was examined by BCA assay kit (Beyotime). Subsequently, $50 \mu \mathrm{g}$ protein was added into 10\% SDS-PAGE and transferred to nitrocellulose filter membranes. Five percent nonfat dried skimmed milk was used to block membranes for $2 \mathrm{~h}$ at room temperature. Membranes were incubated with primary antibodies overnight at $4^{\circ} \mathrm{C}$. The next day, membranes were incubated with secondary antibodies for $1 \mathrm{~h}$ at room temperature and were finally visualized with Odyssey ${ }^{\circledR}$ Infrared Imaging System (Li-COR, NE, USA). Primary antibodies and dilutions used for this study were as follows: LRRC26 (1:1000, GeneTex, SA, USA) and GAPDH (1:5000, Santa Cruz Biotechnology, CA, USA). 


\section{Cell proliferation assay}

CRC cells $\left(2 \times 10^{3}\right.$ per well) were cultured in 96-well plates, and $10 \mu$ l of CCK-8 (Boster Biological Technology, Co. Ltd, Wuhan, China) was added to each well at $0,24,48,72$ and $96 \mathrm{~h}$ after cell seeding. The cells were then incubated for $3 \mathrm{~h}$, and subsequently the supernatants were removed to new 96-well plates. The absorbance at $450 \mathrm{~nm}$ was examined to assess cell viability. Each experiment was performed at least three-times.

\section{Wound healing}

Cells were plated in sixwell plates $\left(1.5 \times 10^{5}\right.$ cells/well), and a wound healing assay was carried out when the cell confluency reached $80 \%$. Briefly, a wound was made by a straight scratch across the middle of the well; phosphatebuffered saline was used to remove the detached cells. The cells were then incubated in serum-free medium and were photographed at $0 \mathrm{~h}$ and $48 \mathrm{~h}$ after scratching. Each experiment was performed at least three-times.

\section{Transwell assays}

Transwell chambers $(8.0 \mu \mathrm{m}$; Corning, NK, USA) with or without Matrigel (BD Biosciences, NJ, USA) were used to assess cell migration and invasion, respectively. Cells were cultured in the top chamber $\left(1 \times 10^{5}\right.$ cells/well $)$ in $100 \mu \mathrm{l}$ of serum-free RPMI 1640; meanwhile, $600 \mu \mathrm{l}$ of RPMI 1640 containing 10\% FBS was added into the lower part of the transwell chamber. After $48 \mathrm{~h}$, invading cells were fixated in $4 \%$ paraformaldehyde for $30 \mathrm{~min}$ and then stained with crystal violet and counted under a microscope. The average number of invading cells in five randomly selected fields was calculated. Each experiment was performed at least three-times.

\section{Statistical analysis}

Statistical analysis was performed by R studio (version 3.6.2) and GraphPad Prism (version 6.0). Two-tailed Student's t-test was used to compare the differences in expression levels between groups. The correlations between expression levels and clinicopathologic parameters were determined using the $\chi^{2}$-test. The permutation test was used for GSEA. Overall survival (OS) curves were generated by Kaplan-Meier analysis and compared with the $\log$-rank test. The $\mathrm{p} \leq 0.05$ was considered to be statistically significant.

\section{Results}

\section{Identification of SNV genotypes in CRC}

MutSigCV was used to predict cancer-related genes in 367 CRC samples, and 81 candidate genes with a significance threshold of $\mathrm{q}<0.05$ were identified (Supplementary Table 1). We then divided the mutations into 96 types according to the bases that were located $1 \mathrm{bp}$ upstream and downstream of the mutation sites and determined the frequency distribution of these mutation types in the 367 CRC samples (Figure 1A). At last, we assessed the frequency of the 96 mutation types in each sample through non-negative matrix decomposition and extracted three mutational signatures (Figure 1B). We analyzed the similarity between these three mutation signatures and COSMIC signatures through $\mathrm{R}$ package maftools. The result illustrated that these mutation signatures were similar to signature 6,1 and 10 in COSMIC, with cosine similarity 0.961, 0.924 and 0.977 respectively (Supplementary Table 2), of which, COSMIC signature 6 and signature 1 are associated with KRAS Wild-Type Cetuximab Resistant CRC [16].

To study the heterogeneity of the mutation characteristics, we compared the distribution of each mutation characteristic in the different CRC samples and performed unsupervised hierarchical clustering. The results illustrated CRC samples were divided into three subgroups based on different mutation characteristics (Figure 1C \& D \& Supplementary Table 3). Then we explored whether there were some differences in clinical features among these three subgroups and statistical analysis of the subtypes' clinical characteristics found significant differences in the stage distribution $(\mathrm{p}=0.01252), \mathrm{N}$ in TNM classification $(\mathrm{p}=0.0003446)$ and tumor location (colon/rectum) $(\mathrm{p}=0.0008531)$. These results indicated that there were correlations between the mutation type and clinical characteristics in CRC (Figure 1E \& Supplementary Table 4).

\section{Comprehensive analysis of CNV-disrupted genes \& DNA methylation in CRC}

The CNV data of the CRC samples were analyzed by GISTIC. A total of 18 amplifications and 26 loss copies of minimal common regions were detected (Supplementary Table 5). Figure 2A \& B show that 13q12.2 and $8 \mathrm{q} 24.21$ were the most significant amplified regions, and $16 \mathrm{p} 13.3$ and 8p21.3 were the most significant loss 


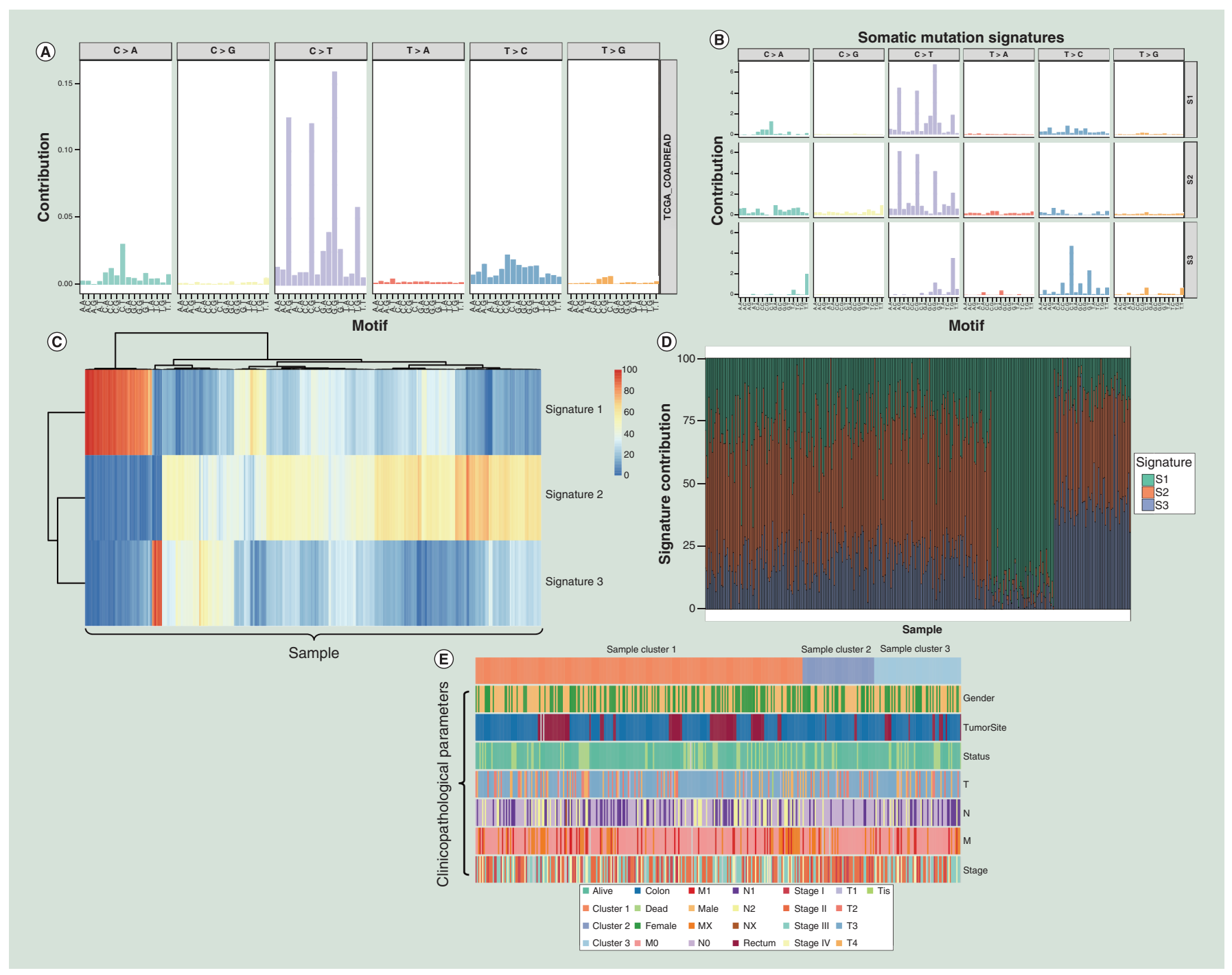

Figure 1. Somatic mutation analysis in colorectal cancer. (A) The landscape of somatic mutation in colorectal cancer (CRC). (B) Non-negative matrix decomposition extracted three mutational signatures. (C) Unsupervised cluster analysis for the proportion of mutational signatures. (D) Distribution of three mutational signatures in CRC. (E) Distribution of clinical features in three subgroups of CRC.

TCGA: The Cancer Genome Atlas.

regions. Unsupervised hierarchical clustering of the CNV results from these samples divided the CRC samples into two subgroups, one of which had obvious CNV changes (Figure 2C \& Supplementary Table 6).

Next, we compared the classification of SNP data with that of CNV data and found that the classification results from the different platforms were consistent. Most of the samples of CNV subgroup 1 were categorized into SNP subgroup 1, and SNP subgroup 2 was categorized into CNV subgroup 2 (Figure 2D \& Supplementary Table 7).

Furthermore, we downloaded 367 CRC samples and 45 normal samples from the TCGA database to screen the differentially methylated sites. We screened out 2719 differentially methylated sites with $p \leq 0.05$ and $|\log F C| \geq 0.4$. Hierarchical cluster analysis of differential DNA methylation sites in all CRC samples and normal samples showed that these methylation sites were clustered into two subgroups (Figure 2E \& Supplementary Table 8). A total of 424 genes were annotated on the 2719 differentially methylated sites, of which 41 genes were differentially expressed (Supplementary Tables $9 \& 10$ ). Annotation information for all methylation sites was shown in Supplementary Table 11.

We also screened out methylation sites of each gene's promoter and gene body, calculated the mean level of these methylation sites and explored their relationship with gene expression through Pearson correlation analysis, 


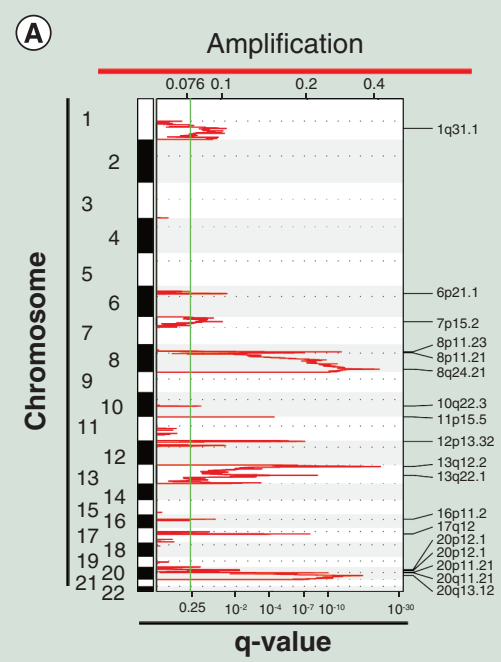

(C)
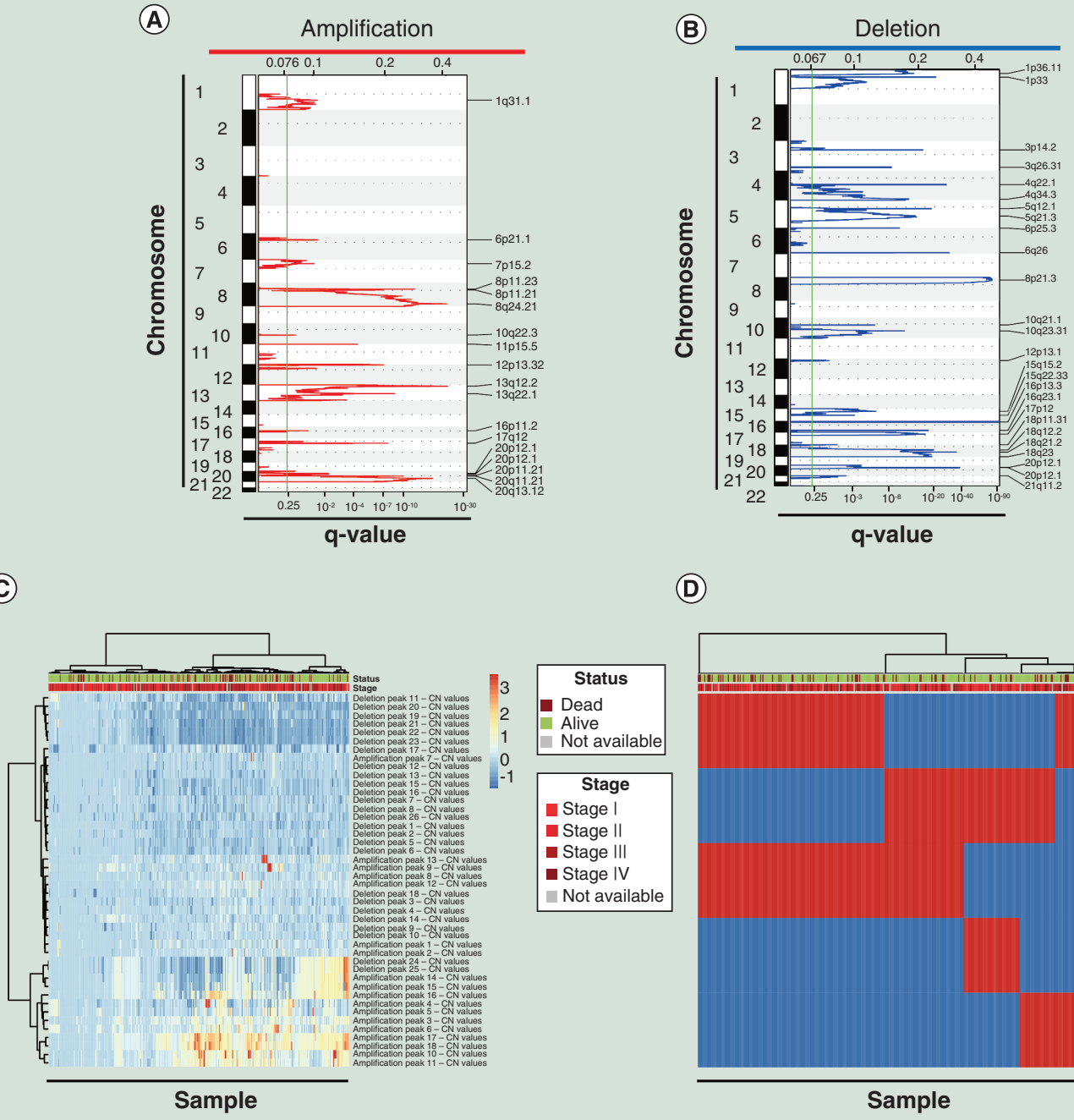

(D)
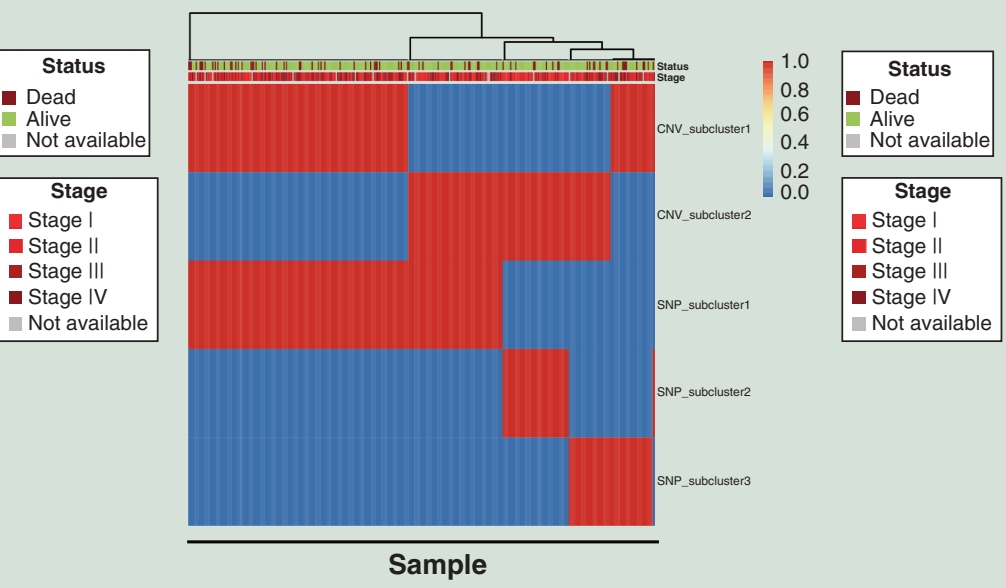

(E)

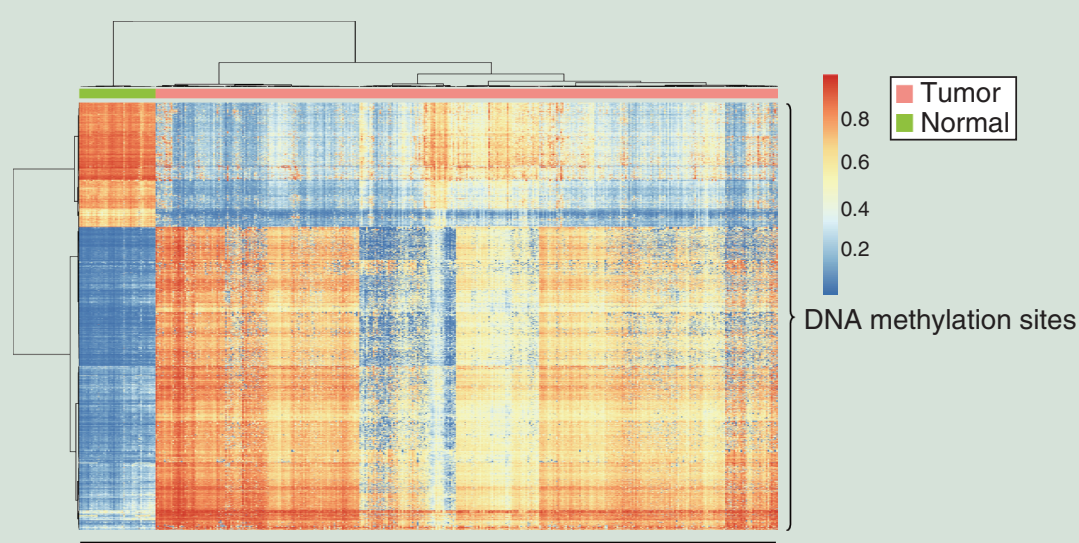

Sample

Figure 2. Copy number variation and DNA methylation analysis in somatic cells. The distribution of (A) copy number variant (CNV) amplification and (B) CNV deletion. (C) Unsupervised hierarchical clustering analysis of CNV. (D) Comparison of classification results from single-nucleotide variant platform and CNV platform. (E) Hierarchical cluster analysis of differential DNA methylation sites. 
the result was shown in Supplementary Table 12. Supplementary Table 13 illustrated the correlation between 41 DEGs and their methylation levels.

Integrative analysis of SNVs, CNVs, DNA methylation \& DEGs that carry CRC risk

A total of 418 samples (comprising 367 CRC specimens and 51 normal specimens) with mRNA expression data were obtained from TCGA, and 1763 genes were identified as DEGs with FDR correction (Supplementary Figure 1 A \& Supplementary Table 14). Among these DEGs, 26 DEGs, including MET, VEGFA, MYC, AXIN2, ETS2, CDK1, ACVRL1, MYH11, FASN, FOXM1, ASNS, LIFR, CAD, NANO, EZH2, LCP1, PHLDA1, NEDD4L, TGIF1, TCF7, FGFR2, RNF43, KIT, B2M, CFH and ABCB1 fell into the previously described consensus molecular subtypes of CRC [17]. Next, we used maftools to estimate the somatic mutagenic influence (including Frame_Shift_Del, Frame_Shift_Ins, In_Frame_Del, Missense_Mutation, Nonsense_Mutation, Nonstop_Mutation, Splice_Site, and Translation_Start_Site) on genes and compared the differences of mutation types between DEGs and non-DEGs. The results showed that the distributions of Missense_Mutation ( $\mathrm{p}=0.03)$ and Nonsense_Mutation $(\mathrm{p}=0.00057)$ mutation types were significantly different between these two types of genes, which indicated that SNVs had a certain influence on gene expression (Supplementary Tables $15 \& 16$ ). Furthermore, a hypergeometric distribution test showed that DEGs were significantly enriched within the significant CRC-related gene set analyzed by MutSigCV ( $\mathrm{p}=0.011)$.

We then obtained CRC-related genes from the NCBI Gene database, the Online Mendelian Inheritance of Man database, and the Kyoto Encyclopedia of Genes and Genomes database (Supplementary Table 17) and identified the overlap between the DEGs and the CRC-related genes. The relationship between DEGs and CRC-related genes is outlined in Supplementary Figure 1B. Three genes were distributed in all four platforms. In addition, 1316 genes were identified only as DEGs, GO analysis showed that they were enriched in biological process such as regulation of cell population proliferation, regulation of cell migration and regulation of cell motility, which might be correlated with CRC tumorigenesis, so these genes may be related to tumorigenesis of CRC (Supplementary Table 18).

At last, to further expand the scope of the investigation, we included in the assessment the 424 genes annotated with differential methylation sites and the 81 significant mutation genes obtained from the MutSigCV analysis. After filtering genes with low expression in most samples, a candidate gene set including 4291 genes was finally obtained (Supplementary Table 19). The expression profiles of these 4291 genes from TCGA were selected for the next step of analysis.

\section{WGCNA analysis to identify hub genes in the key module}

The R package WGCNA was used to create the co-expression network of genes. The clustering was based on the expression data of 4291 genes mentioned above. In sample cluster dendrogram and trait indicators, red color represented survival and white color represented death. We found no outlier samples were detected and removed (Figure 3A). To achieve a scale-free topology $\left(\mathrm{R}^{2}>0.8\right)$, we set $\beta=7$ for the adjacency matrix (Figure 3B). The expression matrix is transformed into adjacency matrix, and then the adjacency matrix is transformed into topology matrix. Based on topological overlap measure, we exploited average linkage hierarchical cluster method to get gene clusters. According to the standard of hybrid dynamic cut tree, we set 20 as minimum gene number for each gene network module. After determining the gene module through the dynamic cutting method, we calculate the eigenvector value of each module, and then cluster the modules, and merge the closer modules into a new module with the height $=0.25$. The results demonstrated that a total of 11 modules were identified (Figure $3 \mathrm{C} \&$ Supplementary Table 20).

In order to determine the correlation between gene modules and disease, we calculated the Pearson's correlation coefficients of each module to sample characteristics and p-value of the corresponding correlation (Supplementary Table 21). Then gene significance value of each module was calculated. Correlation analysis and gene significance value calculation revealed six modules, including brown, blue, pink, red, turquoise and yellow modules were significantly correlated with CRC (Figure 3D).

At last, we constructed a co-expression network for each module and then calculated the degree of each node (Supplementary Table 22). We chose the top 10 genes in each module as the key genes in the co-expression network, and at last, a total of 65 genes changed their expression and co-expressed with other adjacent nodes through interaction, thus affecting downstream biological functions (Supplementary Table 23). Therefore, these genes may be the key genes related to CRC. 


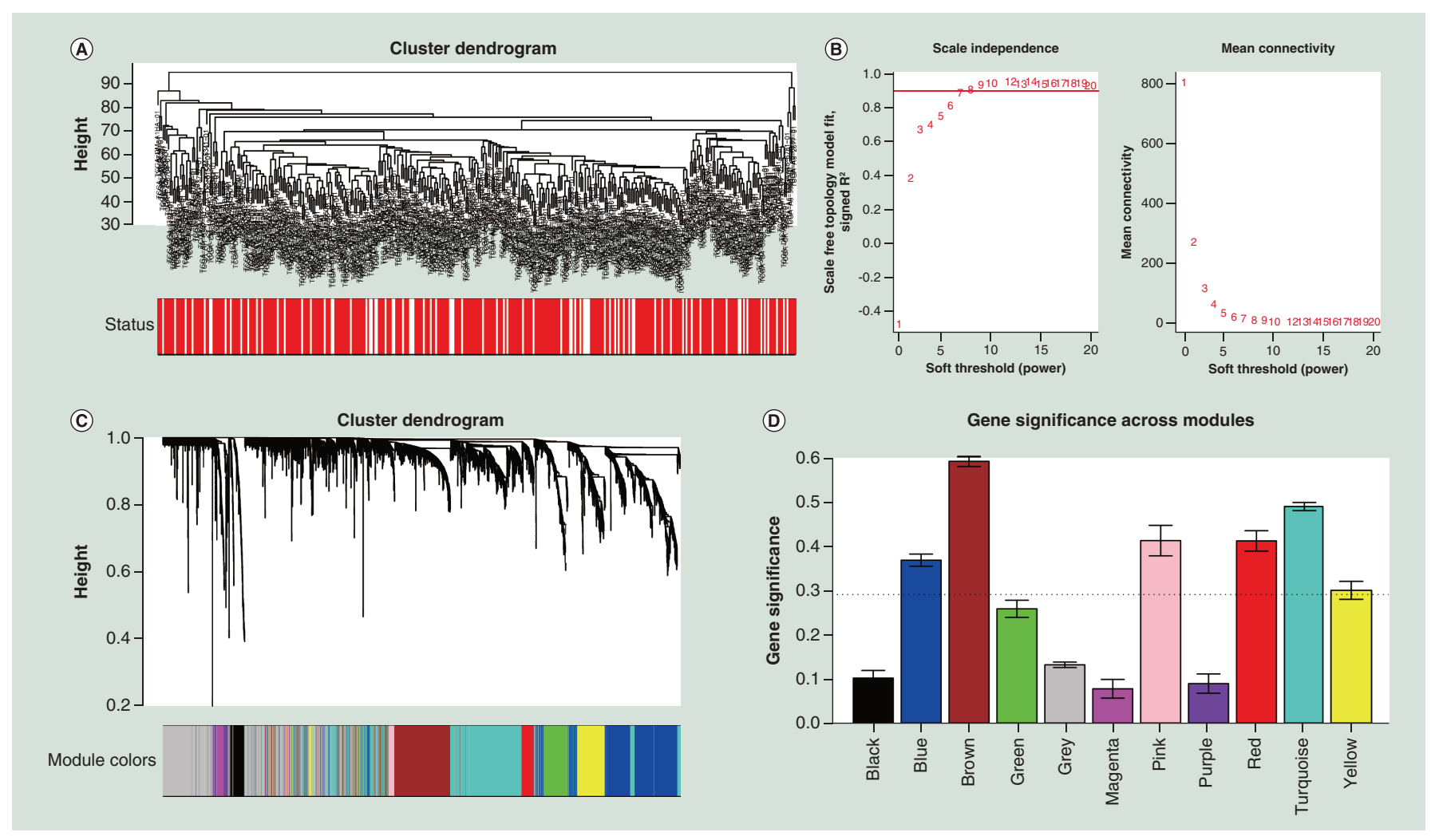

Figure 3. Weighted gene co-expression network analysis to identify hub genes. (A) Dendrogram and trait heatmap of data samples. (B) Determination of parameter $\beta$ of the adjacency function in the weighted gene co-expression network analysis algorithm. (C) Hierarchical clustering dendrogram. (D) Distribution of gene significance in different modules.

Molecular typing of early CRC mediated by driving genes

To determine the prognostic significance of the key CRC-related genes identified in the previous analysis, we downloaded the clinical information of 387 CRC samples from TCGA and performed Kaplan-Meier survival analysis based on the expression of these genes in CRC samples. As shown in Supplementary Table 24, 15 core genes were found to be significantly associated with prognosis. Additionally, we analyzed the prognostic significance of the $81 \mathrm{SNV}$-mutated genes according to their mutations in CRC samples and found that three were prognosisrelated genes, including CCDC73, MBD6 and SMAD2 (Supplementary Table 25).

Next, we selected 187 early-stage CRC samples from TCGA and carried out unsupervised clustering on 18 prognosis-related genes. The results showed that the CRC samples could be divided into two groups (Figure 4A \& B \& Supplementary Tables $26 \& 27$ ). We also analyzed the mutational portrait of the two subtypes, and the results are shown in Figure 4C \& D. Three SNV-mutated genes (CCDC73, MBD6 and SMAD2) were distributed among the two subtypes (Figure 4E \& F); their mutation location is presented in Figure 4G-I.

To further analyze differences between these key genes among the subgroups, the average expression of each gene in each sample was taken as the expression value of the gene. T-test analysis showed that 12 genes (including SPINK4 and REG4) were significantly differentially expressed between the two subgroups (Figure 5A-L \& Supplementary Table 28). Because these 12 genes were screened out for possible variation at a certain dimension, but it does not mean all 12 genes have same variation, so we visualized these 12 genes that differed in mutation, MET and expression spectrum through heat map analysis respectively. Figure $5 \mathrm{M}$ illustrated mutation distribution of candidate genes (NEURL1 did not have any mutations). Figure 5N demonstrated DNA methylation level distribution of candidate genes (NEURL1 and SPINK4 were not screened out any methylation sites). Figure $5 \mathrm{O}$ showed expression distribution of 12 candidate genes and clinicopathological features. 
(A)

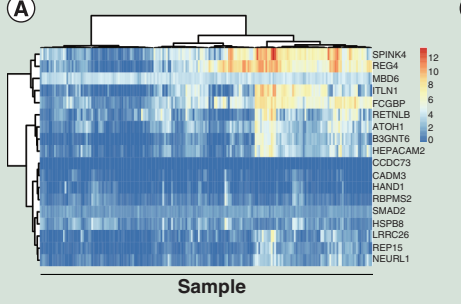

(E)
(B)

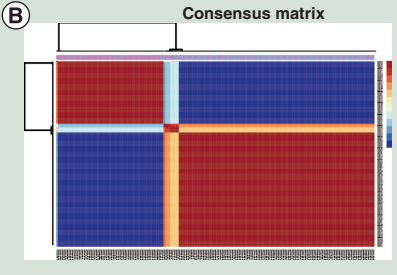

(C)

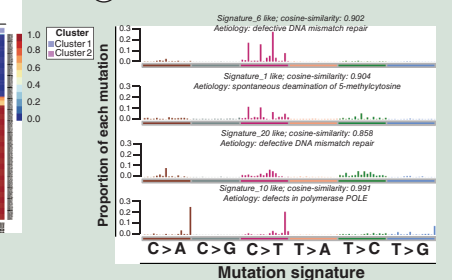

(D)

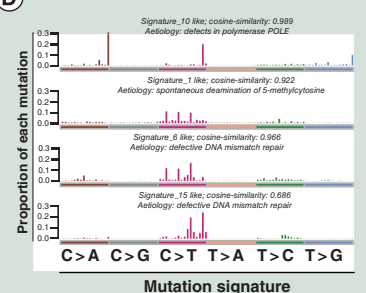

(
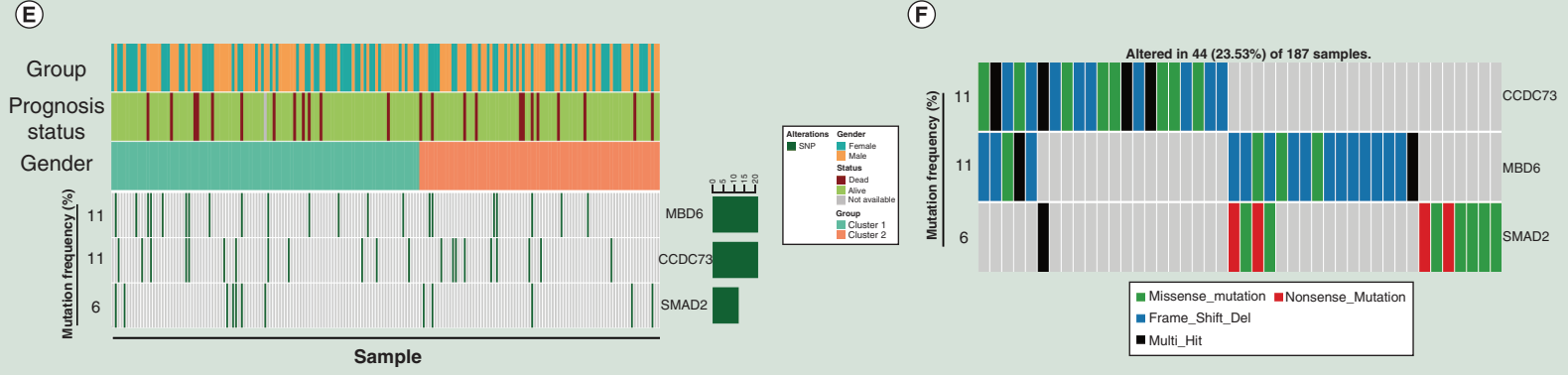

(H)

(1)
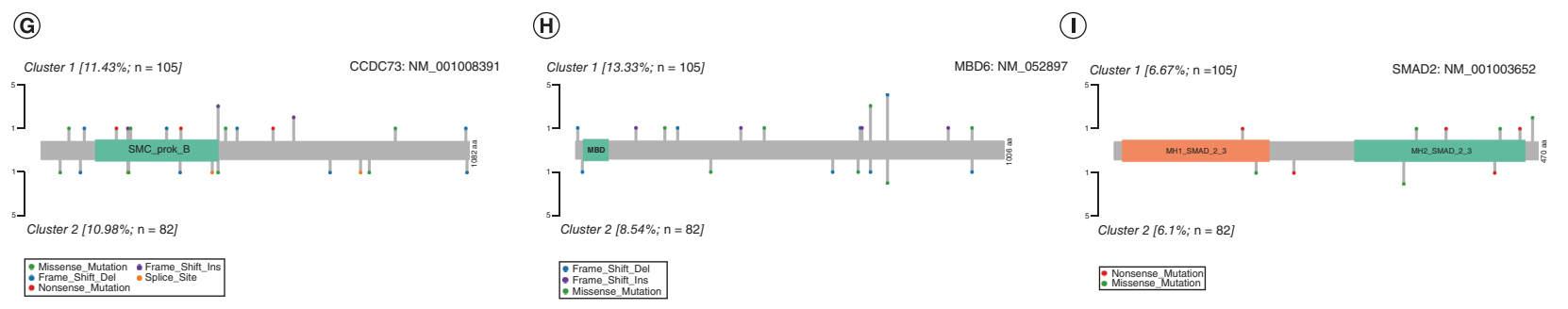

Figure 4. Identification of early-stage colorectal cancer molecular subtypes based on driving genes. (A) Hierarchical cluster analysis of 18 prognosis-related genes in early-stage colorectal cancer (CRC) samples. (B) Non-negative matrix factorization clustering results for 18 prognosis-related genes in early-stage CRC samples. (C \& D) Mutational portrait of early-stage CRC subtypes. (E \& F) The distribution of three single-nucleotide variant-mutated genes (CCDC73, MBD6 and SMAD2) in two subtypes. Mutation location of (G) CCDC73, (H) MBD6 and (I) SMAD2.

\section{Driving genes-associated ceRNA network construction}

Several studies have indicated the significance of noncoding RNA in tumorigenesis [18,19]. In 2011, Salmena et al. put forward their ceRNA hypothesis in which lncRNA was described as an endogenous sponge of microRNA, thereby inhibiting microRNA function. According to this hypothesis, there is a complex regulatory network between lncRNA, microRNA and mRNA [20]. Thus, we sought to construct a ceRNA network for the 12 CRC-related genes.

In the TCGA database, we identified 263 microRNAs and 3886 lncRNAs that were differentially expressed between the CRC and normal samples. We then investigated the co-expression of lncRNAs to find the functional clusters of CRC samples through WGCNA. A soft threshold of $\beta=5$ was chosen to ensure a scale-free network, and this generated six modules-green, turquoise, yellow, brown, blue and red. In addition, the gray module represented a collection of uncorrelated genes (Figure 6A \& B). We further analyzed the correlation between modules and phenotypes to infer the relationships between genes and phenotypes. The integrated analysis results showed that the green, turquoise, brown, blue and red modules were significantly associated with CRC (Figure 6C \& D). We calculated the correlation between gene expression and their modules according to the eigenvectors of each module and chose lncRNAs with R $>0.8$ as key lncRNAs (Supplementary Figures 2-6). A ceRNA network was constructed and visualized using Cytoscape v3.5.0, and it contained 9 CRC-related genes (three genes were excluded because of no significant co-functional noncoding RNA pairs), 31 interacting DEmicroRNAs (20 upregulated and 11 downregulated) and 33 DElncRNAs (5 upregulated and 28 downregulated) (Figure 6E).

Among these elements we identified in this study, some noncoding RNAs were found to be associated with CRC by some previous studies. For example, IncRNA SNHG1 promoted CRC proliferation through sponging a 
(A)

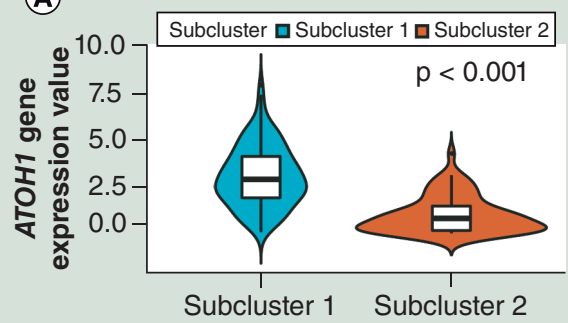

(D)

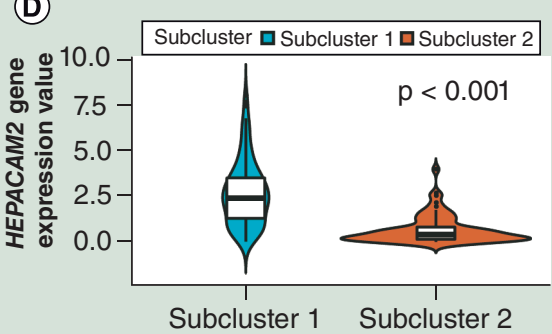

(G)

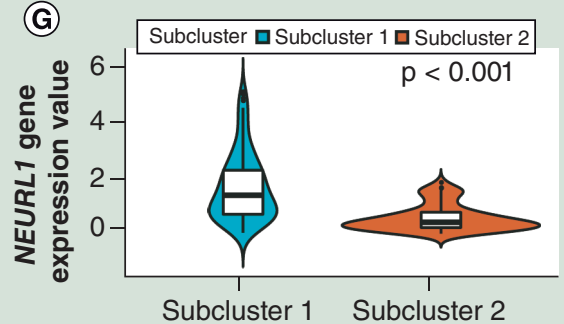

(1)

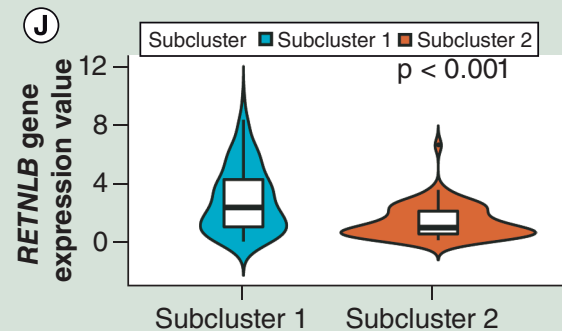

(ii)

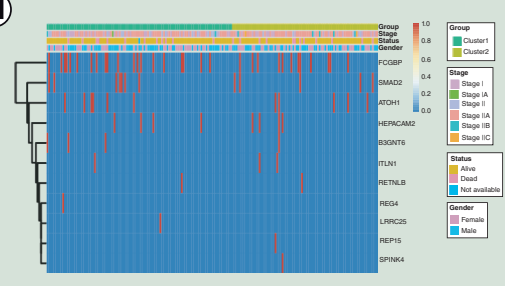

(B)

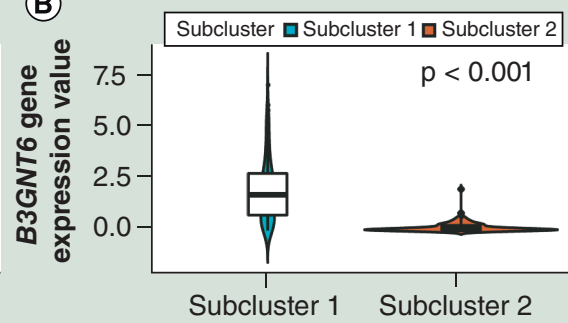

(E)

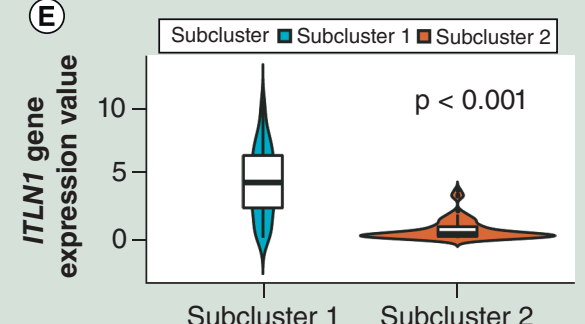

Subcluster 1 Subcluster 2

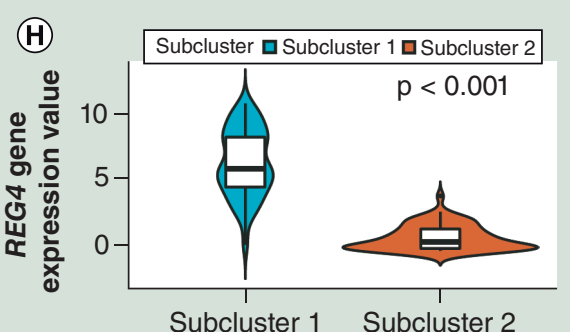

Subcluster 1 Subcluster 2

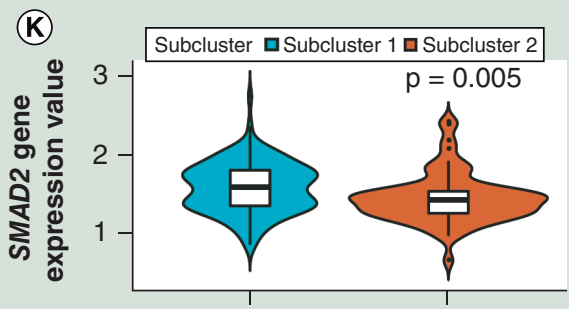

Subcluster 1 Subcluster 2

(1)

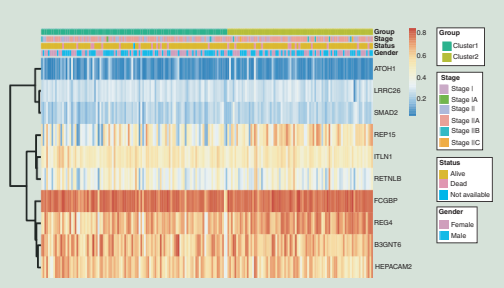

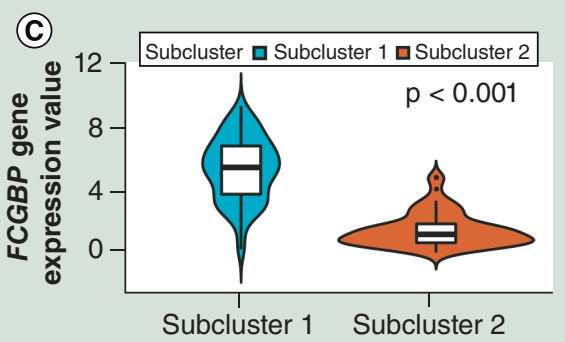

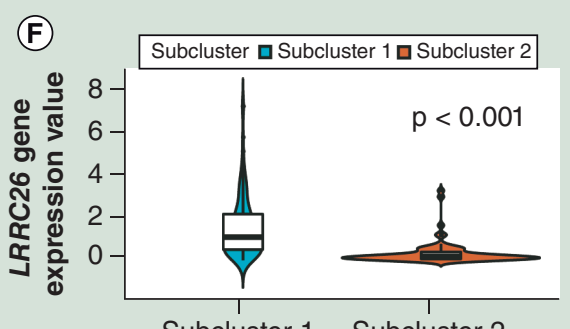

Subcluster 1 Subcluster 2

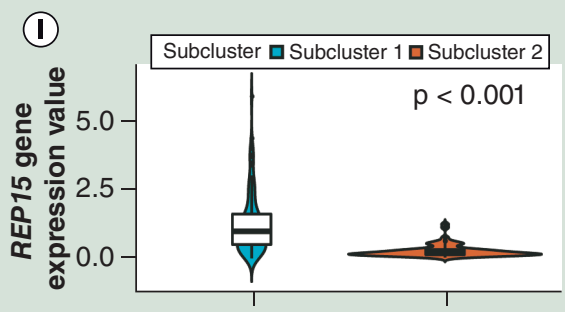

Subcluster 1 Subcluster 2

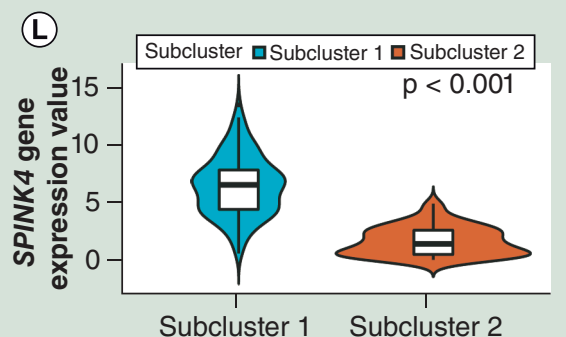

(0)

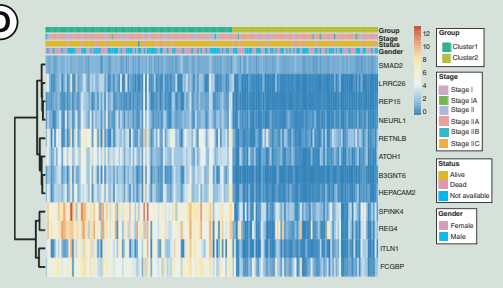

Figure 5. Distribution of driving genes. Gene expression distribution of (A) ATOH1, (B) B3GNT6, (C) FCGBP, (D) HEPACAM2, (E) ITLN1, (F) LRRC26, (G) NEURL1, (H) REG4, (I) REP15, (J) RETNLB, (K) SMAD2 and (L) SPINK4 in two subtypes. (M) Mutation distribution of candidate genes (one gene did not have any mutations). (N) DNA methylation level distribution of candidate genes (two genes did not have any methylation sites). (O) Gene expression distribution of candidate genes and clinicopathological features.

series of microRNAs, including microRNA-137, microRNA-154-5p, and miR-497/miR-195-5p [21-23], Linc00472 was downregulated by DNA hypermethylation in CRC, and overexpressed Linc00472 reduced CRC proliferation by acting as a ceRNA for microRNA-196a, reducing its ability to inhibit PDCD4 expression [24,25]. ZNF667AS1 was found epigenetically-silenced in CRC [26]. HAND2-AS1 inhibits 5-fluorouracil resistance in CRC [27]. Linc02023 suppresses tumorigenesis of CRC in a PTEN-dependent pathway [28]. Downregulated expression of ADAMTS9-AS2 was correlated with the advanced TNM stage and poor histologic differentiation of CRC [29]. 


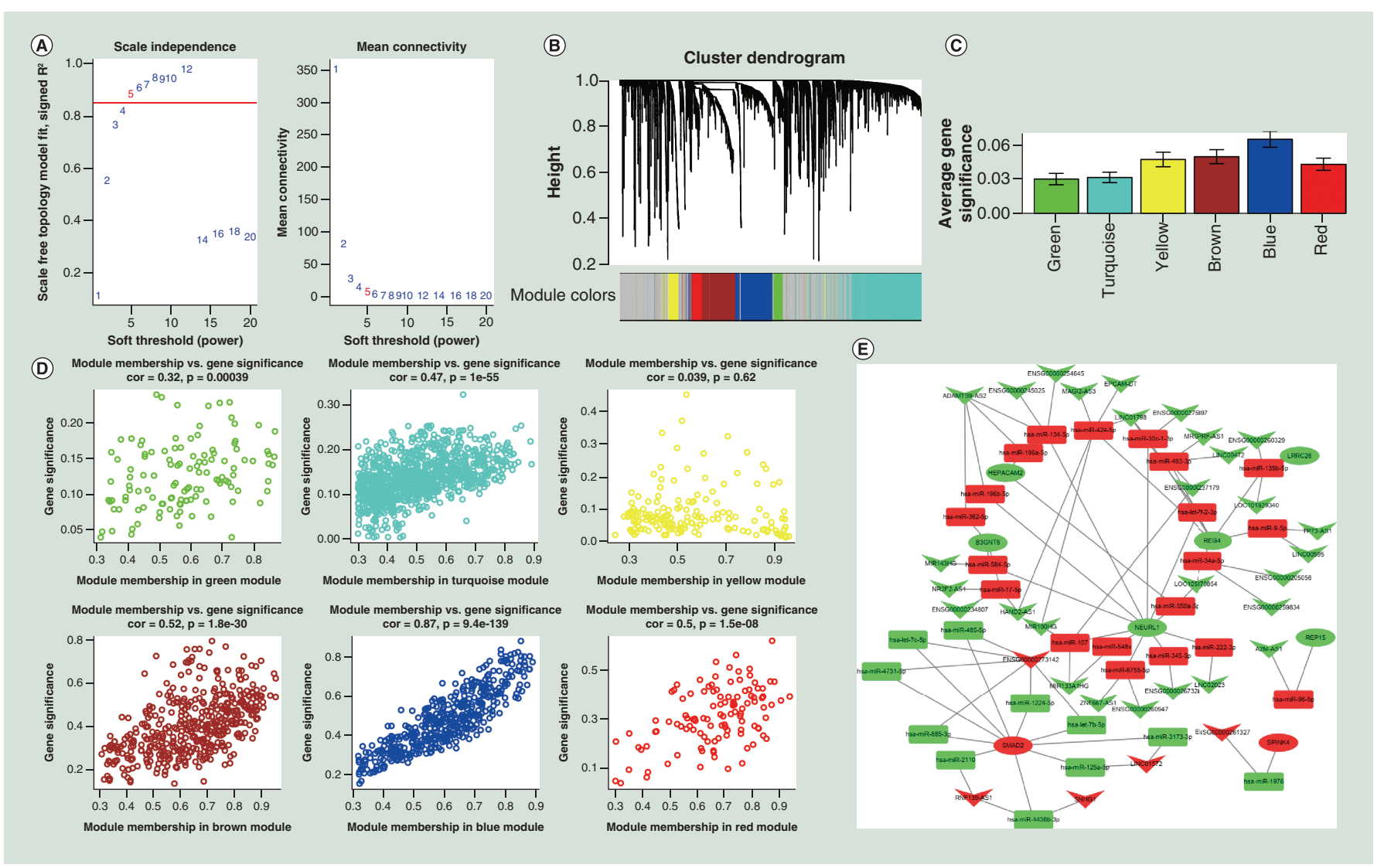

Figure 6. Construction of a driving genes-associated competing endogenous RNA network. (A) Determination of parameter $\beta$ of the adjacency function in the weighted gene co-expression network analysis algorithm. (B) Hierarchical clustering dendrogram. (C) Hierarchical clustering dendrogram. (D) Gene significance in each module. (E) Driving genes-associated competing endogenous RNA network for colorectal cancer.

Some microRNAs such as microRNA-96-5p, let-7c-5p, and microRNA-125a-5p have been demonstrated to be CRCspecific markers for diagnosis and therapy [30-32]. In addition, we exploited survival analysis of these noncoding RNAs in CRC samples of TCGA. The result illustrated that microRNA-2110 (95\% CI: 0.0142-0.0647, $\mathrm{p}=0.002$ ), microRNA-4731-5p (95\% CI: 0.0069-0.9086, $\mathrm{p}=0.047)$, MRGPRF-AS1 (95\% CI: 0.0177-0.0658, $\mathrm{p}=0.006$ ) and ENSG00000267328 (95\% CI: 0.0048-0.0346, p = 0.010) were significantly correlated with OS. In a word, noncoding RNAs that have not been intensively studied may provide new targets for identifying tumorigenesis.

\section{Negative correlation of $L R R C 26$ \& REP15 with CRC aggressiveness}

Among the 12 CRC-related genes, two novel CRC-specific genes, REP15 (NM_001029874.3) and LRRC26 (NM_001013653.2) were demonstrated to be correlated with CRC prognosis; therefore, we sought to assess the prognostic significance of REP15 and LRRC26 in CRC. A total of 172 human CRC samples and their paired adjacent normal tissues were used for validation experiments through immunohistochemistry. We found that CRC patients with high $L R R C 26$ expression were likely have significantly better OS than other groups $(\mathrm{p}=0.027$; Figure 7A). The clinical analysis showed that $L R R C 26$ expression was upregulated in most of paired normal tissues over cancer samples in the $172 \mathrm{CRC}$ cases. Furthermore, LRRC26 immunostaining was positively correlated with the epithelial marker E-cadherin but was negatively correlated with vimentin (Figure 7B). Several clinicopathological parameters were demonstrated to be significantly correlated with LRRC26 expression. High LRRC26 expression was negatively correlated with lymph node metastasis (95\% CI: $0.2703-0.9118, \mathrm{p}=0.023)$, TNM stage $(95 \% \mathrm{CI}$ : $0.2764-0.9531, \mathrm{p}=0.034)$ and distant metastasis (95\% CI: 0.2573-0.9626, $\mathrm{p}=0.037$ ) (Table 1). In addition, high REP15 expression was also present at nontumor tissues, and CRC patients with higher REP15 expression had a better prognosis ( $\mathrm{p}=0.039$, Figure $7 \mathrm{C}$ ), and the staining intensity of REP15 was inversely associated with TNM stage (95\% CI: 0.2631-0.9034, $\mathrm{p}=0.022)$ and distant metastasis (95\% CI: 0.2677-0.9750, $\mathrm{p}=0.040)$ (Table 2). 


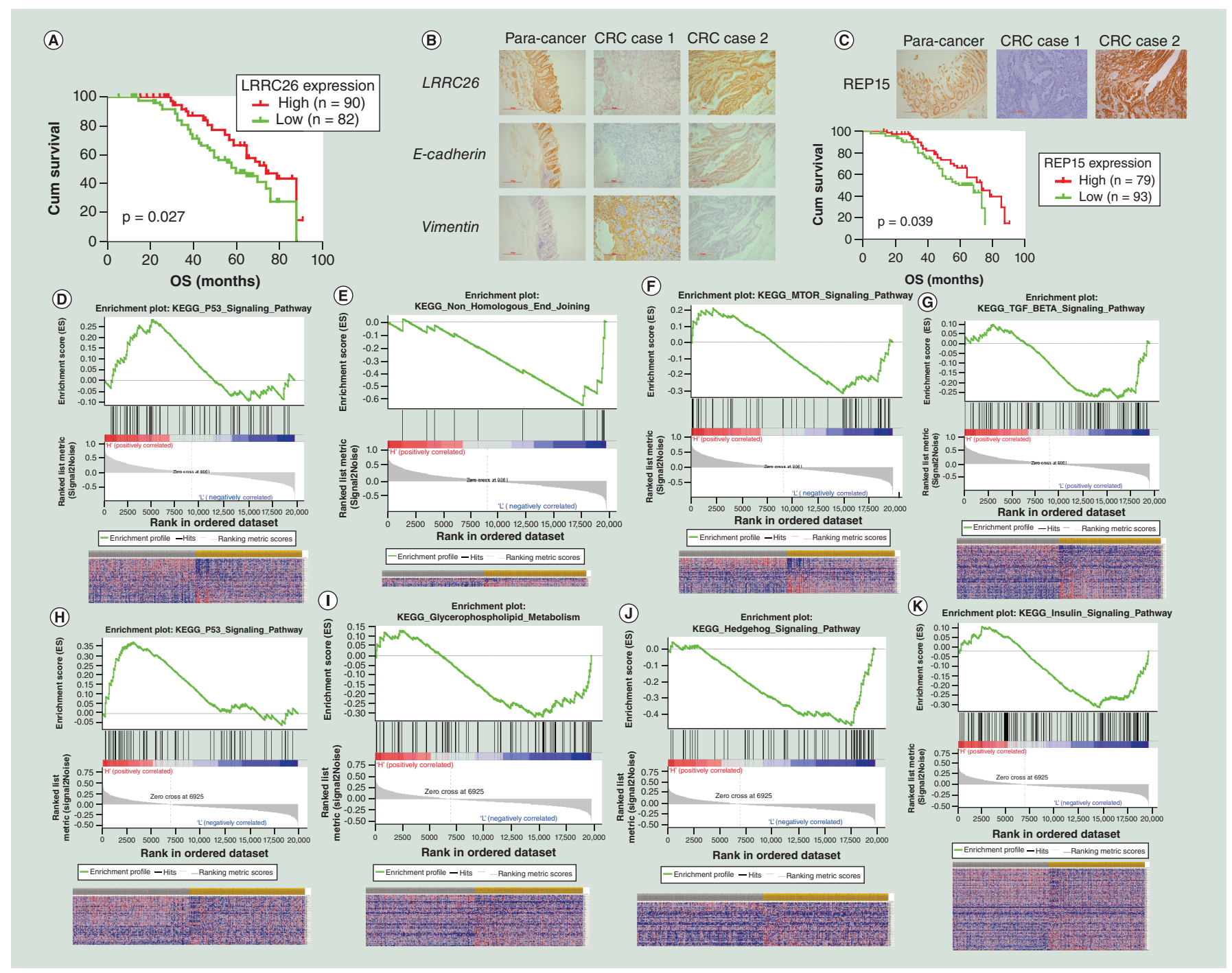

Figure 7. LRRC26 and REP15 acted as tumor suppressers of colorectal cancer. (A) Kaplan-Meier survival analysis on OS of CRC patients with high and low expression levels of $L R R C 26$. (B) Immunohistochemistry staining for $L R R C 26$, E-cadherin and vimentin in CRC samples and adjacent normal tissues. (C) Kaplan-Meier survival analysis on overall survival(OS) of CRC patients with high and low expression levels of REP15. (D-G) GSEA analysis of LRRC26. (H-K) GSEA analysis of REP15.

CRC: Colorectal cancer; GSEA: Gene set enrichment analysis; OS: Overall survival.

Next, we used GSEA to investigate the potential mechanisms of $L R R C 26$ and REP15. The $p 53$ pathway was found to be positively correlated with high $L R R C 26$ expression, and several pathways related to nonhomologous end joining, $m T O R$, and TGF- $\beta$ were negatively correlated with high $L R R C 26$ expression (Figure 7D-G). High REP15 expression was positively correlated with the $p 53$ pathway but negatively correlated with glycerophospholipid metabolism and the hedgehog and insulin pathways (Figure $7 \mathrm{H}-\mathrm{K}$ ). These results indicate a tumor suppressive role for $L R R C 26$ and REP15 in CRC.

To clarify the biological roles of $L R R C 26$ in CRC, we first evaluated the expression of $L R R C 26$ in several CRC cell lines and found that LRRC26 was more highly expressed in HCT116 and Rko cells, and had lower expression in SW480, LoVo, SW620 and HT29 cells (Figure 8A). To examine the potential roles of LRRC26 in CRC tumorigenesis, we overexpressed LRRC26 in SW620 cells and knocked down its expression in Rko cells (Figure 8B). CCK-8 assays showed that LRRC26 hindered cell proliferation (Figure 8C \& D). Wound healing assays showed a reduction in cell motility induced by $L R R C 26$ overexpression (Figure $8 \mathrm{E} \& \mathrm{~F}$ ). Transwell assays demonstrated that LRRC26 significantly decreased the number of migrated and invading CRC cells (Figure 8G 


\begin{tabular}{|c|c|c|c|c|}
\hline \multirow[t]{2}{*}{ Clinical parameter } & \multirow[t]{2}{*}{ Cases } & \multicolumn{3}{|c|}{ LRRC26 expression } \\
\hline & & Low cases & High cases & p-value \\
\hline Tumor location & & & & 0.104 \\
\hline Colon & 77 & 42 & 35 & \\
\hline Rectum & 95 & 40 & 55 & \\
\hline Male & 96 & 46 & 50 & \\
\hline Female & 76 & 36 & 40 & \\
\hline Age & & & & 0.884 \\
\hline$\leq 65$ years & 66 & 31 & 35 & \\
\hline$>65$ years & 106 & 51 & 55 & \\
\hline No & 83 & 47 & 36 & \\
\hline $\mathrm{N} 1 / 2$ & 89 & 35 & 54 & \\
\hline TNM & & & & $0.034^{\dagger}$ \\
\hline$I+I I$ & 101 & 55 & 46 & \\
\hline III + IV & 71 & 27 & 44 & \\
\hline Distant metastasis & & & & $0.037^{\dagger}$ \\
\hline M0 & 116 & 62 & 54 & \\
\hline M1 & 56 & 20 & 35 & \\
\hline
\end{tabular}

$\& \mathrm{H})$. Taken together, these data illustrate that $L R R C 26$ is a negative regulator of proliferation, metastasis and invasion in CRC.

\section{Discussion}

It is well known that $\mathrm{CRC}$ has a complicated carcinogenic progression with variable outcomes; therefore, diagnosis and prognosis in the early stage could help to decrease mortality from CRC. Improvements in microarray and RNA sequencing technologies have made it more convenient to examine mutations and therefore to predict tumor progression, which benefits diagnosis and prognosis evaluation [33,34]. Previous studies have identified a series of CRC-specific genes and noncoding RNAs at the transcriptional and epigenetic levels that may provide important insights for predicting tumorigenesis [35]. However, an increasing number of limitations have been found regarding the identification of pathogenic factors when using a single omics analysis. Recent studies have revealed that multi-omics analysis, which focuses on biological changes at the genomic, epigenomic and transcriptomic levels, can provide insights for tumorigenesis [36]. For example, Chang et al. [37] integrated and analyzed RNA sequence and somatic CNV data to demonstrate dosage-sensitive genes in CRC, which provided a new reference for cancer therapy. In this study, we investigated genes associated with CRC incidence and prognosis. By applying multi-omics analysis, we screened CRC prognostic-related genes and validated their prognostic significance in clinical samples. We downloaded multi-omics data from TCGA and identified significant mutation genes, differential methylation sites and DEGs. We then mapped the DEGs to certain CRC-related genes from the NCBI Gene database, the Online Mendelian Inheritance of Man database, and the Kyoto Encyclopedia of Genes and Genomes database. To obtain the candidate gene sets, the key gene sets were further expanded by adding the significant mutation genes from the mutation data analysis and the genes annotated with differential methylation sites. In addition, WGCNA was used to construct weighted co-expression networks to further mine the hub genes. At last, genes related to prognosis were identified as candidate genes, and the samples of early CRC were classified according to them. Hence, we were able to infer the pathogenesis of CRC by comparing different genes and their functions in 
Table 2. Relationship between REP15 expression and clinicopathological characteristics of colorectal cancer patients.

\begin{tabular}{|c|c|c|c|c|}
\hline \multirow[t]{2}{*}{ Clinical parameter } & \multirow[t]{2}{*}{ Cases } & \multicolumn{3}{|c|}{ REP15 expression } \\
\hline & & Low cases & High cases & $p$-value \\
\hline Number of patients & 172 & 93 & 79 & \\
\hline Tumor location & & & & 0.3 \\
\hline Colon & 77 & 45 & 32 & \\
\hline Rectum & 95 & 48 & 47 & \\
\hline Gender & & & & 0.557 \\
\hline Age & & & & 0.829 \\
\hline$\leq 65$ years & 66 & 35 & 31 & \\
\hline$>65$ years & 106 & 58 & 48 & \\
\hline Tumor size & & & & 0.539 \\
\hline $\mathrm{N} 1 / 2$ & 89 & 42 & 47 & \\
\hline TNM & & & & $0.022^{\dagger}$ \\
\hline$I+I I$ & 101 & 62 & 39 & \\
\hline $\mathrm{III}+\mathrm{IV}$ & 71 & 31 & 40 & \\
\hline Distant metastasis & & & & $0.040^{\dagger}$ \\
\hline Mo & 116 & 69 & 47 & \\
\hline M1 & 56 & 24 & 32 & \\
\hline
\end{tabular}

different clusters. Among the 12 candidate genes, LRRC26 and REP15 were demonstrated to be novel CRC-specific biomarkers.

REP15 (i.e., Rab15 effector protein) was first identified as a binding element to sustain the function of Rab15GTP [38]. Endogenous REP15 is expressed at low levels in HeLa cells, and the overexpression of REP15 reduces the level of transferrin receptor (TfR) on the cell surface, which indicates that REP15 might act as a tumor suppresser via regulating TfR trafficking [39]. Consistent with previous studies, we found that CRC patients with low REP15 expression levels had an unfavorable prognosis, and REP15 expression was inversely correlated with tumor malignancy.

LRRC26, also known as CAPC, was first identified from a cDNA library from breast and prostate cancer cell lines [40,41]. Several studies have demonstrated that LRRC26 functions as a regulatory subunit of BK channels to mediate a variety of biological processes, such as secretory function and the myogenic tone of intravascular pressure [42,43]. Breast, prostate and colon tissues have high LRRC26 expression. LRRC26 expression was present in prostate cancer line LNCaP, but not in poor differentiated prostate cancer lines PC3 and DU145 cells, which indicated an antigen role of $L R R C 26$ in cell differentiation [44]. In addition, downregulation of $L R R C 26$ expression at the transcriptional or epigenetic level promotes malignant behaviors of breast cancer by activating NF- $\kappa \mathrm{B}$ [44,45]. In 2018, Miyagawa et al. [45] demonstrated a frequent methylation in LRRC26 promoter through quantitative polymerase chain reaction and bisulfite pyrosequencing analyses, and the expression of $L R R C 26$ was silenced in TNBC tissues and cell lines. In our current study, we illustrated a somatic mutation of LRRC26 (Supplementary Table 1), and $L R R C 26$ expression was upregulated in most of paired normal tissues over cancer samples. We also found that $L R R C 26$ was positively correlated with $p 53$ pathway but was negatively correlated with nonhomologous end joining, $m T O R$ and TGF- $\beta$ pathway, which indicated a tumor suppressive role of LRRC26. Validation experiments demonstrated that $L R R C 26$ acted as a tumor suppresser of CRC and might oppose the malignant behavior of CRC through attenuation of the epithelial-mesenchymal transition. 
(A)

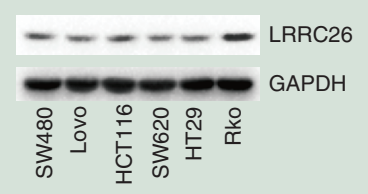

(E)

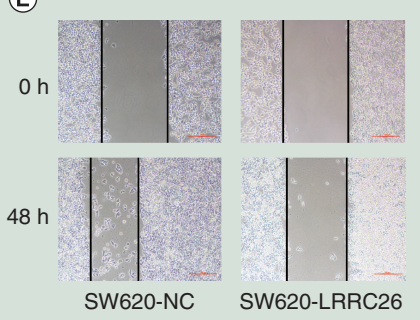

(G)

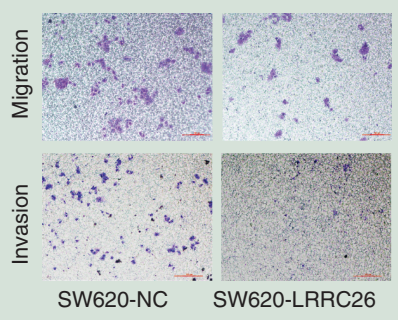

(B)
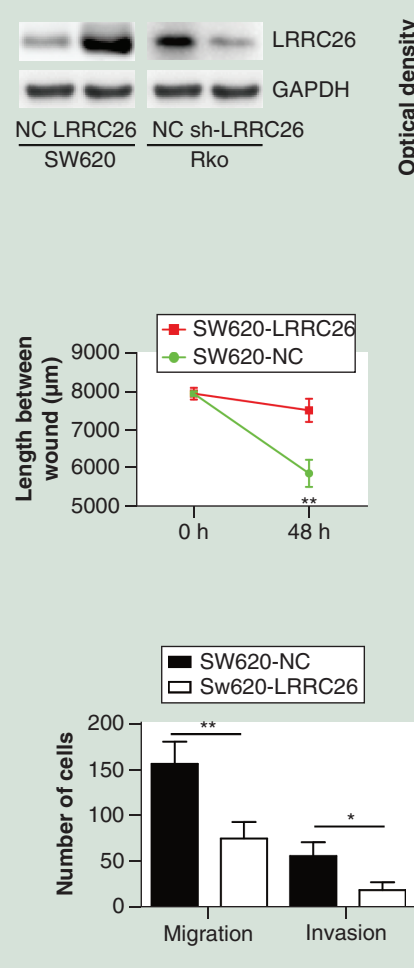

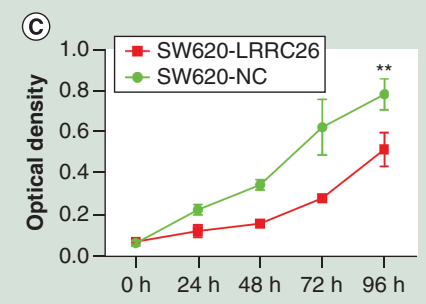

(F)
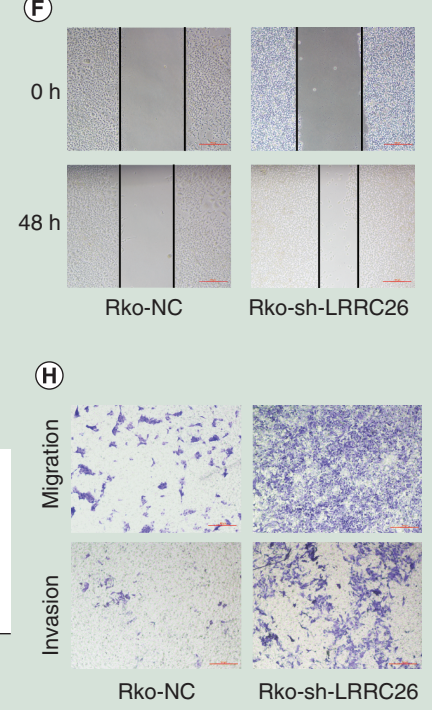
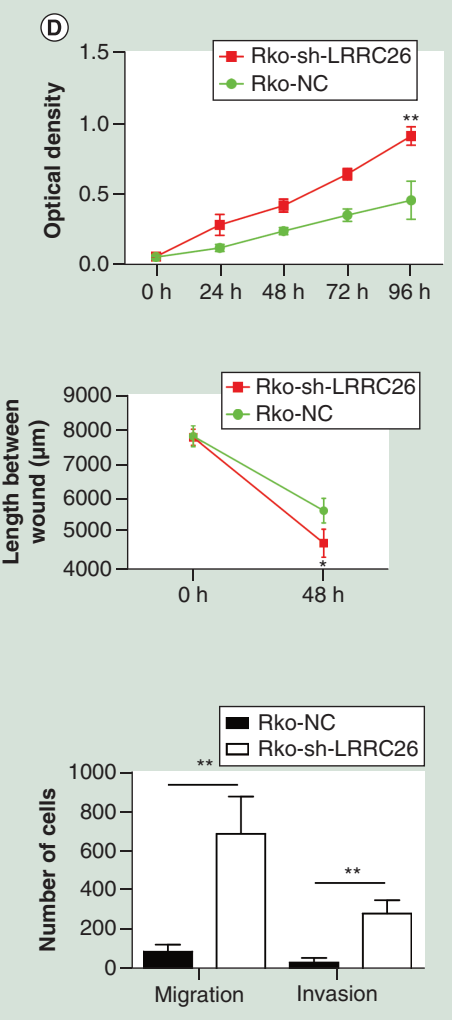

Figure 8. LRRC26 hindered tumorigenesis of colorectal cancer in vitro. (A) $L R R C 26$ expression in CRC cell lines. (B) Overexpression and knockdown of $L R R C 26$ in SW620 and Rko cells, respectively. (C \& D) CCK-8 assays for SW620-NC/SW620-LRRC26 and Rko-NC/Rko-sh-LRRC26 cells. (E \& F) Wound healing assays for SW620-NC/SW620-LRRC26 and Rko-NC/Rko-sh-LRRC26 cells. (G \& H) Transwell motility assays for SW620-NC/SW620-LRRC26 and Rko-NC/Rko-sh-LRRC26 cells.

${ }^{*} \mathrm{p}<0.05 ;{ }^{* *} \mathrm{p}<0.01$.

CRC: Colorectal cancer.

Mutations in genes and noncoding RNAs are often associated with tumor progression. Recent studies indicate that lncRNAs act as microRNA sponges through microRNA-response elements, thereby regulating downstream genes [20,46-49]. In this study, we sought to construct an IncRNA-microRNA-mRNA-ceRNA network to reveal potential crosstalk between candidate genes. We found that 31 interacting DEmicroRNAs and 33 DElncRNAs were involved in the network. Among these elements, some noncoding RNAs were found to be associated with CRC, which support the accuracy of our forecast. Hence, noncoding RNAs that have not been intensively studied may provide new targets for identifying tumorigenesis and we will explore the role of potential candidate noncoding RNAs in CRC tumorigenesis in further study.

The strength of the present study lies in the application of multi-omics to mine CRC-related genes, and we demonstrated LRRC26 and REP15 to be novel CRC-specific genes. Functionally, the in vitro experiments illustrated the tumor-suppressive role of $L R R C 26$. To our knowledge, this is the first report of the prognostic value of LRRC26 and REP15 in CRC, and we demonstrated the biological role of LRRC26 in CRC tumorigenesis. Our study highlights the value of novel CRC-related genes and potential regulatory noncoding RNA networks in CRC. Methods aimed at detecting these elements may provide new strategies for improving CRC diagnosis and prognosis.

\section{Conclusion}

Our research focused on mining driving genes through multi-omics analysis, and we constructed ceRNA network for driving genes. We demonstrated LRRC26 and REP15 as novel CRC-specific driving genes. Functionally, in vitro experiments illustrated the tumor-suppressive role of LRRC26. Our study highlighted the value of novel driving 
genes and potential regulatory noncoding RNA network in CRC, and methods aimed at detecting these elements may provide a new strategy for improving CRC diagnosis and prognosis.

\section{Future perspective}

In the case of mutation analysis, the application of WES or WGS and the depth of the analyses could influence the type of results since the sensitivity can vary according to the limit of detection (for example, in subclonal analyses 'deeper' studies are needed). However, we could not get the original sequencing data, so we could not know the sequencing depth of TCGA data. Besides, although we demonstrated the biological role of $L R R C 26$ and $R E P 15$, their functional and mechanistic aspects were not explored in detail. In addition, we constructed a ceRNA network for the CRC-related genes but did not confirm the regulation patterns of IncRNA, microRNA and mRNA within the ceRNA network. Therefore, mutation and expression profiling with certain sequencing depth of clinical CRC samples would have been a supplement for our study, and additional studies to elucidate the underlying mechanisms should be carried out, and we believe that these further studies will provide a better understanding of the mechanisms of CRC progression.

\section{Summary points}

- We identified colorectal cancer (CRC)-specific driving genes through multi-omics analysis of single-nucleotide variants, copy number variations, DNA methylation and differentially expressed genes identified candidate genes that carry CRC risk.

- A total of 4291 genes were identified as candidate genes through combining differential genes obtained from multicomponent analysis.

- Weighted gene co-expression network analysis identified 65 key genes related to CRC.

- Unsupervised clustering and t-test demonstrated 12 genes as prognosis-related driving genes for molecular typing of early CRC.

- A competing endogenous RNA network for the CRC-related genes was constructed.

- $L R R C 26$ indicated a favor prognosis and high $L R R C 26$ expression was negatively correlated with lymph node metastasis, TNM stage and distant metastasis.

- High REP15 expression indicated a better prognosis, and the staining intensity of REP15 was inversely associated with TNM stage and distant metastasis.

- LRRC26 suppressed malignant behavior of CRC in vitro.

Supplementary data

To view the supplementary data that accompany this paper please visit the journal website at: www.futuremedicine.com/doi/sup $\mathrm{pl} / 10.221 / \mathrm{epi}-2020-0073$

Financial \& competing interest disclosure

This research was supported by grants from the National Natural Science Foundation of China (81702401), the Zhejiang Medical and Health Science and Technology Plan (2019RC175 and 2018KY920), and Taizhou Science and Technology Plan (1902KY191). The authors have no other relevant affiliations or financial involvement with any organization or entity with a financial interest in or financial conflict with the subject matter or materials discussed in the manuscript apart from those disclosed.

No writing assistance was utilized in the production of this manuscript.

Ethical conduct of research

The authors state that they have obtained appropriate institutional review board approval or have followed the principles outlined in the Declaration of Helsinki for all human or animal experimental investigations. In addition, for investigations involving human subjects, informed consent has been obtained from the participants involved. The study was performed with the approval of the Ethics Committee of Sanmen People's Hospital of Zhejiang Province.

Open access

This work is licensed under the Attribution-NonCommercial-NoDerivatives 4.0 Unported License. To view a copy of this license, visit http://creativecommons.org/licenses/by-nc-nd/4.0/ 


\section{References}

Papers of special note have been highlighted as: $\bullet$ of interest; $\bullet \bullet$ of considerable interest

1. Ferlay J, Soerjomataram I, Dikshit R et al. Cancer incidence and mortality worldwide: sources, methods and major patterns in GLOBOCAN 2012. Int. J. Cancer 136(5), E359-E386 (2015).

2. Yuan W, Li X, Liu L et al. Comprehensive analysis of lncRNA-associated ceRNA network in colorectal cancer. Biochem. Biophys. Res. Commun. 508(2), 374-379 (2019).

3. Brenner H, Kloor M, Pox CP. Colorectal cancer. Lancet 383(9927), 1490-1502 (2014).

4. Zheng M, Hu Y, Gou R et al. Integrated multi-omics analysis of genomics, epigenomics, and transcriptomics in ovarian carcinoma. Aging 11(12), 4198-4215 (2019).

5. He Q, He Q, Liu X et al. Genome-wide prediction of cancer driver genes based on SNP and cancer SNV data. Am. J. Cancer Res. 4(4), 394-410 (2014).

6. Fernandez-Rozadilla C, Cazier JB, Tomlinson I et al. A genome-wide association study on copy-number variation identifies a 11q11 loss as a candidate susceptibility variant for colorectal cancer. Hum. Genet. 133(5), 525-534 (2014).

7. Yang Z, Liu B, Lin T, Zhang Y, Zhang L, Wang M. Multiomics analysis on DNA methylation and the expression of both messenger RNA and microRNA in lung adenocarcinoma. J. Cell. Physiol. 234(5), 7579-7586 (2019).

8. Mishra NK, Southekal S, Guda C. Survival analysis of multi-omics data identifies potential prognostic markers of pancreatic ductal adenocarcinoma. Front. Genet. 10, 624 (2019).

9. Rappoport N, Shamir R. Multi-omic and multi-view clustering algorithms: review and cancer benchmark. Nucleic Acids Res. 46(20), 10546-10562 (2018).

10. Lawrence MS, Stojanov P, Polak P et al. Mutational heterogeneity in cancer and the search for new cancer-associated genes. Nature 499(7457), 214-218 (2013).

11. Mayakonda A, Lin DC, Assenov Y, Plass C, Koeffler HP. Maftools: efficient and comprehensive analysis of somatic variants in cancer Genome Res. 28(11), 1747-1756 (2018).

12. Gehring JS, Fischer B, Lawrence M, Huber W. SomaticSignatures: inferring mutational signatures from single-nucleotide variants. Bioinformatics 31(22), 3673-3675 (2015).

13. Ritchie ME, Phipson B, Wu D et al. limma powers differential expression analyses for RNA-sequencing and microarray studies. Nucleic Acids Res. 43(7), e47 (2015).

-• Provides $\mathrm{R}$ package 'limma' that is used to analyze the differences in expression levels/methylation levels.

14. Zhang B, Horvath S. A general framework for weighted gene co-expression network analysis. Stat. Appl. Genet. Mol. Biol. 4(1), Article 17 (2005).

-• Provides weighted gene co-expression network analysis that is performed to determine key genes.

15. Subramanian A, Tamayo P, Mootha VK et al. Gene set enrichment analysis: a knowledge-based approach for interpreting genome-wide expression profiles. Proc. Natl Acad. Sci. USA 102(43), 15545-15550 (2005).

16. Jing C, Wang T, Ma R et al. New genetic variations discovered in KRAS wild-type cetuximab resistant chinese colorectal cancer patients. Mol. Carcinog. 59(5), 478-491 (2020).

17. Guinney J, Dienstmann R, Wang X et al. The consensus molecular subtypes of colorectal cancer. Nat. Med. 21(11), 1350-1356 (2015).

18. Caley DP, Pink RC, Trujillano D, Carter DR. Long noncoding RNAs, chromatin, and development. Sci. World J. 10, 90-102 (2010).

19. Slack FJ, Chinnaiyan AM. The role of non-coding RNAs in oncology. Cell 179(5), 1033-1055 (2019).

20. Salmena L, Poliseno L, Tay Y, Kats L, Pandolfi PP. A ceRNA hypothesis: the Rosetta Stone of a hidden RNA language? Cell 146(3), 353-358 (2011).

- Highlights the theory of competing endorgenous RNA.

21. Xu M, Chen X, Lin K et al. The long noncoding RNA SNHG1 regulates colorectal cancer cell growth through interactions with EZH2 and miR-154-5p. Mol. Cancer 17(1), 141 (2018).

22. Fu Y, Yin Y, Peng $S$ et al. Small nucleolar RNA host gene 1 promotes development and progression of colorectal cancer through negative regulation of miR-137. Mol. Carcinog. 58(11), 2104-2117 (2019).

23. Bai J, Xu J, Zhao J, Zhang R. IncRNA SNHG1 cooperated with miR-497/miR-195-5p to modify epithelial-mesenchymal transition underlying colorectal cancer exacerbation. J. Cell. Physiol. 235(2), 1453-1468 (2020).

24. Ye Y, Yang S, Han Y et al. Linc00472 suppresses proliferation and promotes apoptosis through elevating PDCD4 expression by sponging miR-196a in colorectal cancer. Aging 10(6), 1523-1533 (2018).

25. Chen L, Zhang W, Li DY et al. Regulatory network analysis of LINC00472, a long noncoding RNA downregulated by DNA hypermethylation in colorectal cancer. Clin. Genet. 93(6), 1189-1198 (2018).

26. Vrba L, Garbe JC, Stampfer MR, Futscher BW. A lincRNA connected to cell mortality and epigenetically-silenced in most common human cancers. Epigenetics 10(11), 1074-1083 (2015). 
27. Jiang Z, Li L, Hou Z et al. LncRNA HAND2-AS1 inhibits 5-fluorouracil resistance by modulating miR-20a/PDCD4 axis in colorectal cancer. Cell. Signal. 66, 109483 (2020).

28. Wang Q, Feng Y, Peng W et al. Long noncoding RNA Linc02023 regulates PTEN stability and suppresses tumorigenesis of colorectal cancer in a PTEN-dependent pathway. Cancer Lett. 451, 68-78 (2019).

29. Pan H, Pan J, Song S, Ji L, Lv H, Yang Z. Identification and development of long non-coding RNA-associated regulatory network in colorectal cancer. J. Cell. Mol. Med. 23(8), 5200-5210 (2019).

30. Li J, Chen Y, Guo X et al. GPC1 exosome and its regulatory miRNAs are specific markers for the detection and target therapy of colorectal cancer. J. Cell. Mol. Med. 21(5), 838-847 (2017).

31. Zhou XG, Huang XL, Liang SY et al. Identifying miRNA and gene modules of colon cancer associated with pathological stage by weighted gene co-expression network analysis. Onco. Targets Ther. 11, 2815-2830 (2018).

32. Gattolliat $\mathrm{CH}$, Uguen A, Pesson $\mathrm{M}$ et al. MicroRNA and targeted mRNA expression profiling analysis in human colorectal adenomas and adenocarcinomas. Eur. J. Cancer 51(3), 409-420 (2015).

33. Damin F, Galbiati S, Soriani N et al. Analysis of KRAS, NRAS and BRAF mutational profile by combination of in-tube hybridization and universal tag-microarray in tumor tissue and plasma of colorectal cancer patients. PLoS ONE 13(12), e0207876 (2018).

34. Kabir MF, Mohd Ali J, Haji Hashim O. Microarray gene expression profiling in colorectal (HCT116) and hepatocellular (HepG2) carcinoma cell lines treated with Melicope ptelefolia leaf extract reveals transcriptome profiles exhibiting anticancer activity. PeerJ 6, e5203 (2018)

35. Yiu AJ, Yiu CY. Biomarkers in Colorectal Cancer. Anticancer Res. 36(3), 1093-1102 (2016).

36. Hasin Y, Seldin M, Lusis A. Multi-omics approaches to disease. Genome Biol. 18(1), 83 (2017).

-. A comprehensive review regarding the importance of multi-omics analysis.

37. Chang Z, Miao X, Zhao W. Identification of prognostic dosage-sensitive genes in colorectal cancer based on multi-omics. Front. Genet. 10,1310 (2019).

38. Elferink LA, Strick DJ. Functional properties of rab15 effector protein in endocytic recycling. Methods Enzymol. 403, 732-743 (2005).

39. Strick DJ, Elferink LA. Rab15 effector protein: a novel protein for receptor recycling from the endocytic recycling compartment. Mol. Biol. Cell. 16(12), 5699-5709 (2005).

40. Egland KA, Vincent JJ, Strausberg R, Lee B, Pastan I. Discovery of the breast cancer gene BASE using a molecular approach to enrich for genes encoding membrane and secreted proteins. Proc. Natl Acad. Sci. USA 100(3), 1099-1104 (2003).

41. Egland KA, Liu XF, Squires $S$ et al. High expression of a cytokeratin-associated protein in many cancers. Proc. Natl Acad. Sci. USA 103(15), 5929-5934 (2006).

42. Yang C, Gonzalez-Perez V, Mukaibo T, Melvin JE, Xia XM, Lingle CJ. Knockout of the LRRC26 subunit reveals a primary role of LRRC26-containing BK channels in secretory epithelial cells. Proc. Natl Acad. Sci. USA 114(18), E3739-E3747 (2017).

43. Evanson KW, Bannister JP, Leo MD, Jaggar JH. LRRC26 is a functional BK channel auxiliary gamma subunit in arterial smooth muscle cells. Circ. Res. 115(4), 423-431 (2014).

44. Liu XF, Xiang L, Zhang Y, Becker KG, Bera TK, Pastan I. CAPC negatively regulates NF-kappaB activation and suppresses tumor growth and metastasis. Oncogene 31(13), 1673-1682 (2012).

45. Miyagawa $Y$, Matsushita $Y$, Suzuki $\mathrm{H}$ et al. Frequent downregulation of $L R R C 26$ by epigenetic alterations is involved in the malignant progression of triple-negative breast cancer. Int. J. Oncol. 52(5), 1539-1558 (2018).

46. Wee LM, Flores-Jasso CF, Salomon WE, Zamore PD. Argonaute divides its RNA guide into domains with distinct functions and RNA-binding properties. Cell 151(5), 1055-1067 (2012).

47. Li X, Ao J, Wu J. Systematic identification and comparison of expressed profiles of IncRNAs and circRNAs with associated co-expression and ceRNA networks in mouse germline stem cells. Oncotarget 8(16), 26573-26590 (2017).

48. Tay Y, Rinn J, Pandolfi PP. The multilayered complexity of ceRNA crosstalk and competition. Nature 505(7483), 344-352 (2014).

49. Qu L, Ding J, Chen C et al. Exosome-transmitted lncARSR promotes sunitinib resistance in renal cancer by acting as a competing endogenous RNA. Cancer Cell 29(5), 653-668 (2016). 